\title{
The nature of the 8 o'clock arc using Near-IR IFU spectroscopy with SINFONI
}

\author{
M. Shirazi ${ }^{1}$, S. Vegetti ${ }^{2}$, N. Nesvadba ${ }^{3}$, J. Brinchmann ${ }^{1}$, S. Allam ${ }^{4}$, \\ and D. Tucker ${ }^{4}$ \\ ${ }^{1}$ Leiden Observatory, Leiden University, P.O. Box 9513, 2300 RA Leiden, The Netherlands \\ email: shirazi@strw.leidenuniv.nl \\ ${ }^{2}$ Kavli Institute for Astrophysics and Space Research, Massachusetts Institute of Technology, \\ Cambridge, MA 02139, USA \\ ${ }^{3}$ Institut d'Astrophysique Spatiale, UMR 8617, CNRS, Université Paris-Sud, Bâtiment 121, \\ 91405, Orsay Cedex, France \\ ${ }^{4}$ Fermi National Accelerator Laboratory, P.O. Box 500, Batavia, IL 60510, USA
}

We present an analysis of the lensed Lyman Break Galaxy (LBG), the 8 o'clock arc, at redshift 2.735. We reduced Near-IR IFU data from SINFONI on VLT covering $\lambda=2900$ $\AA$ to $6500 \stackrel{\circ}{A}$ in the rest-frame. From this we recovered the $\mathrm{H} \beta$ map and the spatiallyresolved $\mathrm{H} \beta$ profile which are shown in the right plot in Fig. 1 . We can see that $\mathrm{H} \beta$ shows different profiles at different spatial pixels and it is composed of multiple components. To study the de-lensed morphology of the galaxy we make use of existing $\mathrm{B} \& \mathrm{H}$ band imaging from the HST. Based on this we constructed a rigorous lens model for the system using the Bayesian grid based lens modeling technique presented by Vegetti \& Koopmans (2009, MNRAS, 392, 945). In order to obtain a robust lens model, we first considered the high resolution B band HST image (rest-frame UV, left plot in Fig. 1) and then used this modeling to reconstruct the $\mathrm{H} \beta$ line map of the galaxy. We then present the de-lensed $\mathrm{H} \beta$ line map, velocity and velocity dispersion maps of this LBG galaxy.
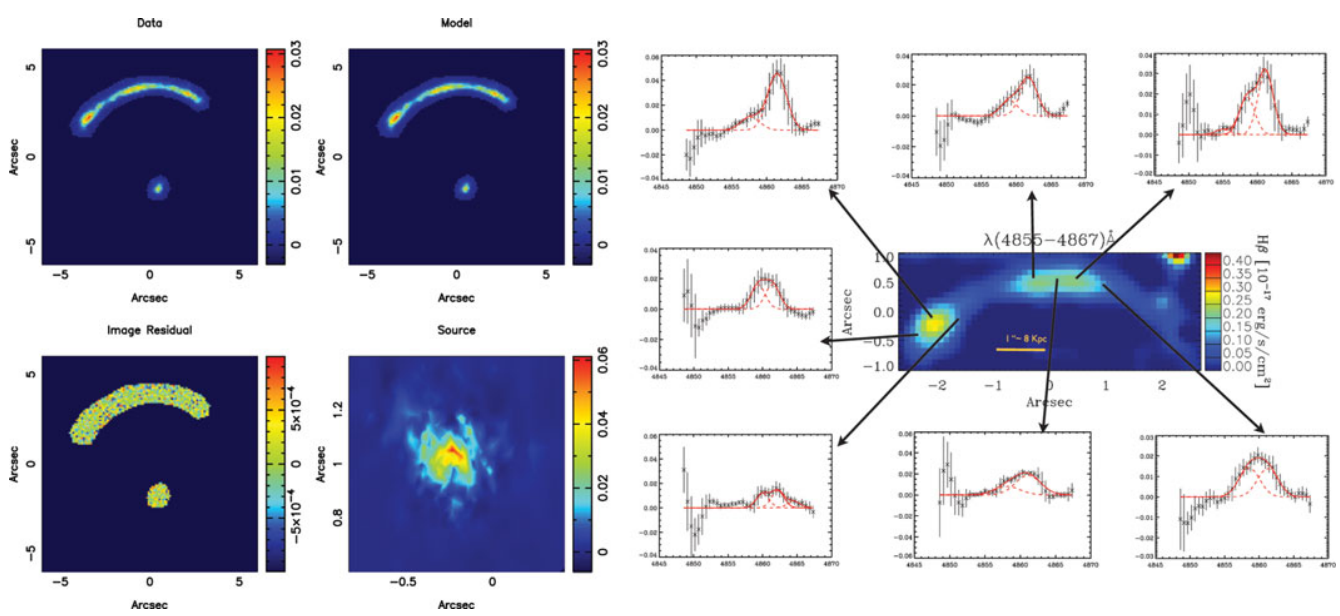

Figure 1. Left plot: The left top panel shows the arc and the counter image in the B band HST image. The elliptical has been removed from this image.The right top panel shows the best-fit model and the next panel shows the residuals after subtracting this model from the data. The reconstructed image is shown on the right bottom panel. From this image we can see that the source is formed of multiple components, two main galaxy components and a clump separated by 0.15 arcsec. Right plot: The spatially-resolved asymmetric $\mathrm{H} \beta$ profiles of the arc and the $\mathrm{H} \beta$ map are shown. The $\mathrm{H} \beta$ map is integrated between the wavelengths mentioned in the map. 